\title{
Bipartite graphs with at most six non-zero eigenvalues
}

\author{
Mohammad Reza Oboudi * \\ Department of Mathematics, College of Sciences, Shiraz University, \\ Shiraz, 71457-44776, Iran \\ School of Mathematics, Institute for Research in Fundamental Sciences (IPM), \\ P.O. Box 19395-5746, Tehran, Iran
}

Received 22 October 2014, accepted 13 November 2015, published online 2 January 2016

\begin{abstract}
In this paper we characterize all bipartite graphs with at most six non-zero eigenvalues. We determine the eigenvalues of bipartite graphs that have at most four non-zero eigenvalues.
\end{abstract}

Keywords: Eigenvalues of graphs, bipartite graphs.

Math. Subj. Class.: 05C31, 05C50

\section{Introduction and Main Results}

Throughout this paper we will consider only simple graphs. A graph with no isolated vertices is called reduced (also called canonical in the literature) if no two non-adjacent vertices have the same neighborhood. It is well known that the number of vertices of reduced graphs are bounded by some functions of the rank, see [7]. It is not hard to see if a reduced graph has exactly $m$ non-zero eigenvalues, then $G$ has at most $2^{m}-1$ vertices. Therefore the number of non-isomorphic reduced graphs with a given number $m$ of nonzero eigenvalues ( counted by their multiplicities) is finite. So it is natural to look for a classification of reduced graphs with a given number of non-zero eigenvalues. Note that the number of non-zero eigenvalues of a graph is equal to the rank of the graph which by definition is the rank of the adjacency matrix of the graph. It is not hard to characterize the reduced graphs with rank $r$, for $r \leq 3$. The classification of reduced graphs of rank 4 and rank 5 can be found in [8] and also in [1] and [2], respectively. Thus it is natural to classify all reduced graphs of rank 6 ( see [8] for a characterization according to minimal subgraphs

* This research was in part supported by a grant from IPM (No. 94050012).
E-mail address: mr_oboudi@yahoo.com (Mohammad Reza Oboudi) 
of nullity one). Recently, in [9], triangle-free graphs of rank 6 are claimed to be classified in Theorem 4.1. But we find that the graphs $G_{2}$ and $G_{3}$ in the main result in [9], have rank 8 . On the other hand, the main step to find a classification for triangle-free graphs of rank 6 is first to classify bipartite graphs of the same rank (see [9]). In this paper, in a different way from [5] and [9], we completely characterize all bipartite graphs with rank $r$, where $r \in\{2,4,6\}$. In other words, we find all bipartite graphs with at most six non-zero eigenvalues. Also we find the eigenvalues of bipartite graphs of rank 4 .

\section{Notation and Preliminaries}

Let $G=(V, E)$ be a graph. The order of $G$ denotes the number of vertices of $G$. For two graphs $G_{1}=\left(V_{1}, E_{1}\right)$ and $G_{2}=\left(V_{2}, E_{2}\right)$, the disjoint union of $G_{1}$ and $G_{2}$ denoted by $G_{1}+G_{2}$ is the graph with vertex set $V_{1} \cup V_{2}$ and edge set $E_{1} \cup E_{2}$. The graph $r G$ denotes the disjoint union of $r$ copies of $G$. Let $u$ and $v$ be two vertices of a graph $G$. By $u v$ we mean an edge between $u$ and $v$. For every vertex $v \in V$, the open neighborhood of $v$ is the set $N(v)=\{u \in V: u v \in E\}$. An independent set in a graph is a set of pairwise non-adjacent vertices. For every vertex $v \in V(G)$, the degree of $v$ that is denoted by $\operatorname{deg}(v)$ is the number of edges incident with $v$. We denote by $K_{n}, K_{m, n}, C_{n}$, and $P_{n}$, the complete graph of order $n$, the complete bipartite graph with part sizes $m, n$, the cycle of order $n$, and the path of order $n$, respectively.

If $\left\{v_{1}, \ldots, v_{n}\right\}$ is the set of vertices of a graph $G$, then the adjacency matrix of $G$, $A(G)=\left(a_{i j}\right)$, is an $n \times n$ matrix where $a_{i j}$ is 1 if $v_{i}$ is adjacent to $v_{j}$, otherwise $a_{i j}=0$. Thus $A(G)$ is a symmetric matrix with zeros on the diagonal and all the eigenvalues of $A(G)$ are real. By $[v]$ we mean the column of $A(G)$ that correspond to a vertex $v$ of $G$, that is the vector of neighbors of $v$. By the eigenvalues of $G$ we mean those of its adjacency matrix. The multiset of eigenvalues of $G$ is denoted by $\operatorname{Spec}(G)$. We let $\operatorname{Spec}(G)=$ $\left\{\lambda_{1}, \ldots, \lambda_{n}\right\}$, where $\lambda_{1} \geq \cdots \geq \lambda_{n}$ are the eigenvalues of $G$. The rank of $G$, denoted by $\operatorname{rank}(G)$, is the rank of $A(G)$. In fact, $\operatorname{rank}(G)$ is the number of all non-zero eigenvalues of $G$. We consider the rank of graphs over the field of real numbers. Let $H$ be a bipartite graph with parts $X$ and $Y$. Suppose that $X=\left\{u_{1}, \ldots, u_{m}\right\}$ and $Y=\left\{v_{1}, \ldots, v_{n}\right\}$. By $B(G)=\left(b_{i j}\right)$ we mean the $(0,1)$ matrix of size $m \times n$ such that $b_{i j}=1$ if and only if $u_{i}$ and $v_{j}$ are adjacent, otherwise $b_{i j}=0$. We note that the rank of any bipartite graph is even. More precisely:

Remark 2.1. Let $H$ be a bipartite graph with parts $X$ and $Y$. Let $B=B(H)$, as mentioned above. Then we have $\operatorname{rank}(H)=2 \operatorname{rank}(B)$.

Definition 2.2. A graph $G$ is called reduced or canonical if it has no isolated vertex and for any two non-adjacent vertices $u$ and $v, N(u) \neq N(v)$. Equivalently, any row of the adjacency matrix is non-zero and no two rows of the adjacency matrix of $G$ are equal.

Definition 2.3. Let $G$ be a graph. We say $G$ is neighbor-reduced if $G$ has no isolated vertex and there are no three vertices $u, v$ and $w$ satisfying all the following conditions:

1) $\{u, v, w\}$ is an independent set.

2) $N(u) \cap N(v)=\varnothing$.

3) $N(w)=N(u) \cup N(v)$.

Equivalently, any row of the adjacency matrix is non-zero and no row of the adjacency matrix of $G$ is the sum of two other rows. 
Definition 2.4. A graph $G$ is called strongly reduced, if $G$ is reduced and neighbor-reduced.

We note that there is no connection between Definitions 2.2 and 2.3. For example the path $P_{5}$ is reduced but it is not neighbor-reduced while the cycle $C_{4}$ is not reduced but it is neighbor-reduced. The cycle $C_{6}$ is reduced and neighbor-reduced graph, that is $C_{6}$ is strongly reduced. Also the graph $H$ which is shown in Figure 1 is neither reduced (because it has a duplicate vertex) nor neighbor-reduced (because it has $P_{5}$ as an induced subgraph ( Table 1 of [8])).

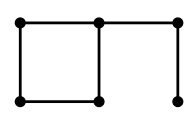

$H$

Figure 1: A non-reduced and non-neighbor-reduced bipartite graph.
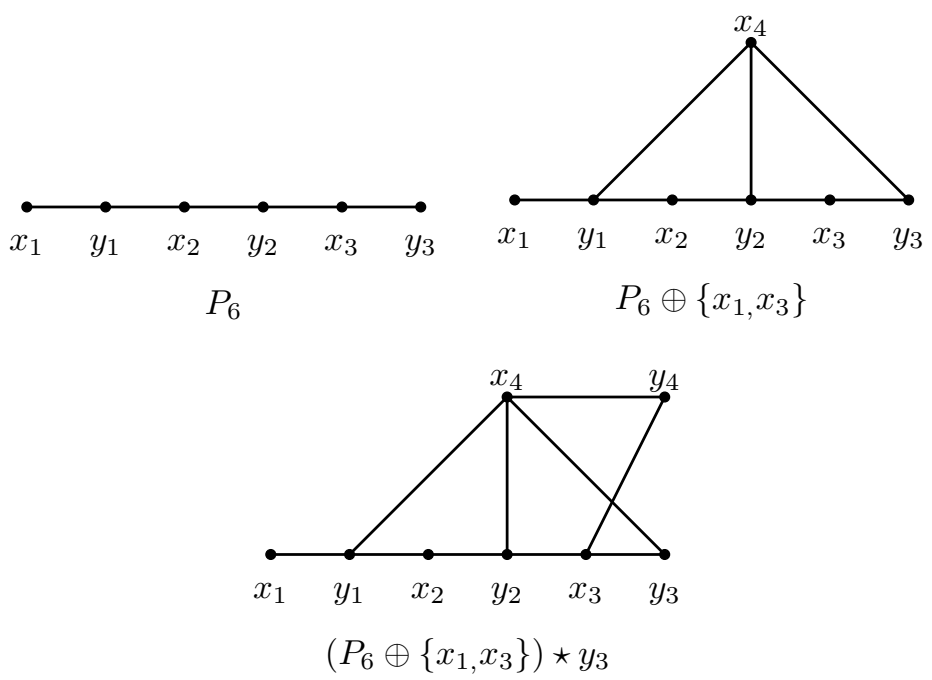

Figure 2: Three bipartite graphs with rank 6 obtained from $P_{6}$ with operations $\star$ and $\oplus$.

Let $G$ be a graph and $v$ be a vertex of $G$. Suppose that $N(v)=\left\{v_{1}, \ldots, v_{k}\right\}$. By $G \star v$, this operation is well known in the literature as adding a duplicate vertex, we mean the graph obtained by adding a new vertex $w$ and new edges $\left\{w v_{1}, \ldots, w v_{k}\right\}$ to $G$. Thus in $G \star v, N(w)=N(v)$, that is $G \star v$ is not reduced (see Figure 2). Let $S$ be an independent set of $G$ which is ordered as $S=\left\{u_{1}, \ldots, u_{t}\right\}$ and $t \geq 2$. We inductively define $G \star S$ as $\left(G \star u_{1}\right) \star S \backslash\left\{u_{1}\right\}$. We note that the operation has the effect of adding even cycles and no odd cycles to the graph; therefore $G$ is bipartite if and only if $G \star v$ is bipartite.

Suppose that $u$ and $v$ are non-adjacent vertices of $G$ such that $N(u) \cap N(v)=\varnothing$. By $G \oplus\{u, v\}$ we mean the graph obtained by adding a new vertex $w$ to $G$ and new edges 
$\{w x: x \in N(u)\} \cup\{w y: y \in N(v)\}$. Thus in $G \oplus\{u, v\}, N(w)=N(u) \cup N(v)$. See Figure 2. It is easy to prove the following theorem:

Theorem 2.5. Let $G$ be a graph and $u$ and $v$ be two non-adjacent vertices of $G$. Then

$$
\operatorname{rank}(G)=\operatorname{rank}(G \star v)=\operatorname{rank}(G \oplus\{u, v\}) .
$$

Theorem 2.5 shows that for classifying the graphs of rank $r$ it is sufficient to classify the reduced graphs ( or the neighbor-reduced graphs) of rank $r$. Thus in sequel we consider the reduced graphs.

\section{Graphs of Rank 2}

In this section we characterize all graphs with rank 2. We note that $\operatorname{rank}(G)=0$ if and only if $G=n K_{1}$, for some natural number $n$. By the fact that the sum of all eigenvalues of a graph is zero, there is no graph with rank 1 .

Remark 3.1. Let $G$ be a graph of rank 2. Thus $\operatorname{Spec}(G)=\{\alpha, 0, \ldots, 0, \beta\}$, for some nonzero real numbers $\alpha$ and $\beta$. Since for any graph $H, \sum_{\lambda \in \operatorname{Spec}(H)}=0$, we obtain $\beta=-\alpha$. Thus $G$ is bipartite ( see Theorem 3.11 of [4]). In the other words, any graph of rank 2 is bipartite.

Theorem 3.2. Let $G$ be a reduced graph. Then $\operatorname{rank}(G)=2$ if and only if $G=P_{2}$.

Proof. Assume that $\operatorname{rank}(G)=2$. By Remark 3.1, $G$ is bipartite. Let $X$ and $Y$ be the parts of $G$ and $B=B(G)$. By Remark 2.1, $\operatorname{rank}(B)=1$. This shows that all columns of $B$ are the same. In the other words, all vertices of part $X$ have the same neighbors. Since $G$ is reduced, we conclude that $|X|=1$. Similarly, $|Y|=1$. Thus $G=P_{2}$.

Theorem 3.3. Let $G$ be a graph. Then $\operatorname{rank}(G)=2$ if and only if $G=K_{m, n}+t K_{1}$, for some natural numbers $m$ and $n$ and a non-negative integer $t$.

Proof. First suppose that $\operatorname{rank}(G)=2$. If $G$ is reduced, then by Theorem 3.2, $G=$ $P_{2}+t K_{1}$, for some $t \geq 0$. Assume that $G$ is not reduced. By deleting some vertices from $G$, one can obtain a reduced graph $H$. By Theorem 2.5, $\operatorname{rank}(H)=2$. Thus by the connected case, $H=K_{2}+t K_{1}$. Since $G=H \star S$, for some independent set $S$ of $G$, we obtain that $G=K_{m, n}+t K_{1}$, for some integers $m, n \geq 1$. The second part of assertion is trivial.

\section{Bipartite Graphs of Rank 4 and their Eigenvalues}

In this section we investigate about the bipartite graphs with rank 4 . We note that by a different method in [5], the bipartite graphs of rank 4 are classified.

Theorem 4.1. Let $G$ be a reduced bipartite graph. Then $\operatorname{rank}(G)=4$ if and only if $G=2 P_{2}$ or $G=P_{4}$ or $G=P_{5}$.

Proof. One can easily see that $\operatorname{rank}\left(2 P_{2}\right)=\operatorname{rank}\left(P_{4}\right)=\operatorname{rank}\left(P_{5}\right)=4$. It remains to prove the other part. Suppose that $G$ is a reduced bipartite graph. Let $X$ and $Y$ be the parts of $G$ and $B=B(G)$. By Remark 2.1, $\operatorname{rank}(B)=2$. Let $u, v \in Y$. Suppose that $[u]$ and $[v]$ are two columns of $B$ correspondence to $u$ and $v$, respectively. Assume that $[u]=\alpha[v]$, 
for some real $\alpha$. It is trivial that $\alpha \in\{0,1\}$. If $\alpha=0$, then $u$ is an isolated vertex, a contradiction. Thus $\alpha=1$. This shows that $N(u)=N(v)$, a contradiction. Therefore very two columns of $B$ are independent.

Let $x_{1}, x_{2}, x_{3}$ be three vertices of $X$. Since $\operatorname{rank}(B)=2$ and any two columns of $B$ are independent, for some non-zero real numbers $a$ and $b$ we have

$$
\left[x_{3}\right]=a\left[x_{1}\right]+b\left[x_{2}\right] .
$$

By multiplying the vector $j$ (all components are 1) to both sides of Equation 4.1, we obtain that:

$$
\operatorname{deg}\left(x_{3}\right)=\operatorname{adeg}\left(x_{1}\right)+b \operatorname{deg}\left(x_{2}\right) .
$$

Since $N\left(x_{1}\right) \neq N\left(x_{2}\right)$, without losing the generality let $N\left(x_{1}\right) \nsubseteq N\left(x_{2}\right)$. Assume that $u \in N\left(x_{1}\right) \backslash N\left(x_{2}\right)$. Considering the entries correspondence to $u$ in the vectors $\left[x_{1}\right],\left[x_{2}\right]$ and $\left[x_{3}\right]$ in the Equality (4.1), we obtain $a \in\{0,1\}$. Since $a$ is non-zero, $a=1$. Let $w$ be a neighbor of $x_{2}$. Considering the entries correspondence to $w$ in the Equality (4.1), we have $b \in\{-1,0,1\}$. Since $b \neq 0, b= \pm 1$. Thus for any three columns $X_{1}, X_{2}$ and $X_{3}$ of $B$, one of the following holds:

$$
X_{1}=X_{2}+X_{3} \quad \text { or } \quad X_{2}=X_{1}+X_{3} \text { or } \quad X_{3}=X_{1}+X_{2} .
$$

We claim that $|X|,|Y| \leq 3$. By contradiction suppose that $|X| \geq 4$. Let $x_{1}, x_{2}, x_{3}, x_{4}$ be the vertices of $X$ with have the minimum degree among all vertices of $X$. Suppose that $\operatorname{deg}\left(x_{1}\right) \geq \operatorname{deg}\left(x_{2}\right) \geq \operatorname{deg}(3) \geq \operatorname{deg}\left(x_{4}\right)$. Since the sets $\left\{\left[x_{1}\right],\left[x_{2}\right],\left[x_{3}\right]\right\}$ and $\left\{\left[x_{2}\right],\left[x_{3}\right],\left[x_{4}\right]\right\}$ are dependent, by Equations 4.2 and 4.3 , we obtain $\left[x_{1}\right]=\left[x_{2}\right]+\left[x_{3}\right]$ and $\left[x_{2}\right]=\left[x_{3}\right]+\left[x_{4}\right]$. Therefore $\left[x_{1}\right]=2\left[x_{3}\right]+\left[x_{4}\right]$, a contradiction (by Equation 4.3). Similarly, $|Y| \leq 3$. Since $|X|,|Y| \leq 3, G$ has at most 6 vertices. It is not hard to see that $G$ is isomorphic to one of the graphs $2 P_{2}$ or $P_{4}$ or $P_{5}$. The proof is complete.

Let $V\left(2 P_{2}\right)=\left\{x_{1}, y_{1}, x_{2}, y_{2}\right\}$ and $E\left(2 P_{2}\right)=\left\{x_{1} y_{1}, x_{2} y_{2}\right\}$. Thus $P_{5}=2 P_{2} \oplus$ $\left\{x_{1}, x_{2}\right\}$. Therefore $P_{5}$ is not neighbor-reduced. Using this fact and Theorem 4.1 we obtain the following:

Theorem 4.2. Let $G$ be a strongly reduced bipartite graph. Then $\operatorname{rank}(G)=4$ if and only if $G=2 P_{2}$ or $G=P_{4}$.

Using Theorems 2.5 and 4.1 ( similar to proof of Theorem 3.3), one can characterize all bipartite graphs of rank 4 .

Theorem 4.3 (Theorem 7.1 of [8]). Let $G$ be a bipartite graph with no isolated vertex. Then $\operatorname{rank}(G)=4$ if and only if $G$ is obtained from $2 P_{2}$ or $P_{4}$ or $P_{5}$ with the operation $\star$.

We finish this section by computing the eigenvalues of bipartite graphs of rank 4 . First we prove the following lemma. For other proofs, see also page 115 of [4], page 54 of [3] and page 115 of [6].

Lemma 4.4. Let $G$ be a bipartite graph. Let $\operatorname{Spec}(G)=\left\{\lambda_{1}, \ldots, \lambda_{n}\right\}$. Then

$$
\lambda_{1}^{4}+\cdots+\lambda_{n}^{4}=2 m+8 q+4 \sum_{v \in V(G)}\left(\begin{array}{c}
\operatorname{deg}(v) \\
2
\end{array}\right),
$$

where $m$ and $q$ are the number of edges and the number of cycles of length 4 of $G$, respectively. 
Proof. It is well-known that for any natural number $k, \lambda_{1}^{k}+\cdots+\lambda_{n}^{k}$ is equal to the number of closed walks of $G$ with length $k$ ( page 81 of [4]). Since $G$ is bipartite, all closed walks of length 4 appear in the subgraphs of $G$ that are isomorphic to $P_{2}, P_{3}$ or $C_{4}$. We note that in any $P_{2}, P_{3}$ and $C_{4}$ there are 2,4 and 8 closed walks of length 4 ( which cover all vertices of $P_{2}, P_{3}$ and $C_{4}$ ), respectively. On the other hand, the number of $P_{3}$ in $G$ is $\sum_{v \in V(G)}\left(\begin{array}{c}\operatorname{deg}(v) \\ 2\end{array}\right)$. This completes the proof.

Theorem 4.5. Let $G$ be a bipartite graph of rank 4 . Let $\operatorname{Spec}(G)=\{\alpha, \beta, 0, \ldots, 0,-\beta$, $-\alpha\}$. Suppose that $v$ is a vertex of $G$ with $\operatorname{deg}(v)=t$. Then $\operatorname{Spec}(G \star v)=\{\gamma, \theta, 0, \ldots, 0$, $-\theta,-\gamma\}$, where $\gamma$ and $\theta$ are as following:

$$
\gamma, \theta=\sqrt{\frac{\alpha^{2}+\beta^{2}+t \pm \sqrt{2\left(\alpha^{4}+\beta^{4}+L\right)-\left(\alpha^{2}+\beta^{2}+t\right)^{2}}}{2}} .
$$

Also,

$$
\gamma, \theta=\sqrt{\frac{m+t \pm \sqrt{2\left(m^{2}-2 \alpha^{2} \beta^{2}+L\right)-(m+t)^{2}}}{2}},
$$

where $L=t+6\left(\begin{array}{l}t \\ 2\end{array}\right)+2 \sum_{w \in N(v)} \operatorname{deg}(w)$.

Proof. Assume that $m$ and $m^{\prime}$ are the number of edges of $G$ and $G \star v$, respectively. Suppose that $q$ and $q^{\prime}$ are the number of cycles of length 4 of $G$ and $G \star v$, respectively. Thus $m^{\prime}=m+t$ and $q^{\prime}=q+\left(\begin{array}{l}t \\ 2\end{array}\right)$. Since $G$ is bipartite, $G \star v$ is bipartite, too. By Theorem 2.5, $\operatorname{rank}(G \star v)=4$. Therefore we have $\operatorname{Spec}(G \star v)=\{\gamma, \theta, 0, \ldots, 0,-\theta,-\gamma\}$, for some non-zero real numbers $\gamma$ and $\theta$. Using Lemma 4.5 we obtain

$$
\begin{aligned}
2\left(\gamma^{4}+\theta^{4}\right)= & 2(m+t)+8\left(q+\left(\begin{array}{l}
t \\
2
\end{array}\right)\right) \\
& +4 \sum_{w \in V(G) \backslash N(v)}\left(\begin{array}{c}
\operatorname{deg}(w) \\
2
\end{array}\right)+4 \sum_{w \in N(v)}\left(\begin{array}{c}
\operatorname{deg}(w)+1 \\
2
\end{array}\right)+4\left(\begin{array}{l}
t \\
2
\end{array}\right) .
\end{aligned}
$$

Since for any integer $k \geq 0,\left(\begin{array}{c}k+1 \\ 2\end{array}\right)=\left(\begin{array}{c}k \\ 2\end{array}\right)+k$, using Lemma 4.5 we conclude that:

$$
\gamma^{4}+\theta^{4}=\alpha^{4}+\beta^{4}+t+6\left(\begin{array}{c}
t \\
2
\end{array}\right)+2 \sum_{w \in N(v)} \operatorname{deg}(w) .
$$

On the other hand, by the fact that for every graph $H, \sum_{\lambda \in \operatorname{Spec}(H)} \lambda^{2}=2|E(H)|$, we obtain that $\alpha^{2}+\beta^{2}=m$ and $\gamma^{2}+\theta^{2}=m+t$. These equalities imply that

$$
\gamma^{2}+\theta^{2}=\alpha^{2}+\beta^{2}+t
$$

Let $L=t+6\left(\begin{array}{l}t \\ 2\end{array}\right)+2 \sum_{w \in N(v)} \operatorname{deg}(w)$. Suppose that $p=\gamma^{2}$ and $q=\theta^{2}$. Using Equations 4.5 and 4.6 we obtain

$$
p q=\frac{\left(\alpha^{2}+\beta^{2}+t\right)^{2}-\left(\alpha^{4}+\beta^{4}+L\right)}{2}, \text { and } p+q=\alpha^{2}+\beta^{2}+t .
$$

The latter equalities show that $p$ and $q$ are roots of the following polynomial:

$$
x^{2}-\left(\alpha^{2}+\beta^{2}+t\right) x+\frac{\left(\alpha^{2}+\beta^{2}+t\right)^{2}-\left(\alpha^{4}+\beta^{4}+L\right)}{2} .
$$

The proof is complete. 
Remark 4.6. Let $f(G, \lambda)$ be the characteristic polynomial of $G$. Suppose that $G$ is a bipartite graph of order $n$ with rank 4 and with $\operatorname{Spec}(G)=\{\alpha, \beta, 0, \ldots, 0,-\beta,-\alpha\}$. Let $m$ be the number of edges of $G$. Thus $f(G, x)=x^{n-4}\left(x^{4}-m x^{2}+\alpha^{2} \beta^{2}\right)$. Assume that $v$ is a vertex of $G$ with $\operatorname{deg}(v)=t$ and $L=t+6\left(\begin{array}{l}t \\ 2\end{array}\right)+2 \sum_{w \in N(v)} \operatorname{deg}(w)$. Then by the proof of Theorem 4.5

$$
f(G \star v, x)=x^{n-3}\left(x^{4}-\left(\alpha^{2}+\beta^{2}+t\right) x^{2}+\frac{\left(\alpha^{2}+\beta^{2}+t\right)^{2}-\left(\alpha^{4}+\beta^{4}+L\right)}{2}\right) .
$$

\section{Bipartite Graphs of Rank 6}

In this section we characterize all bipartite graphs of rank 6 . We note that all the singular graphs of rank 6 are characterized in [8]. In addition the bipartite singular ( reduced) graphs of nullity one are those corresponding to the last four lines of Table 2 in [8], that is the graphs shown in Figure 7 in [8]. These are the graphs $G_{8}$ to $G_{12}$ of our paper. The graphs $G_{1}$ to $G_{7}$ are non-singular and $G_{13}$ has nullity two. As we mentioned in the introduction, in [9] the authors consider the triangle-free graphs of rank 6. But we find that the graphs $G_{2}$ and $G_{3}$ in Theorem 4.1 of [9], have rank 8. On the other hand, the main step to find a classification for triangle-free graphs of rank 6 is first to classify bipartite graphs of the same rank [9]. In this section by a different way from [9], we completely characterize all bipartite graphs of rank 6 . In other words, we find all bipartite graphs with exactly six non-zero eigenvalues.

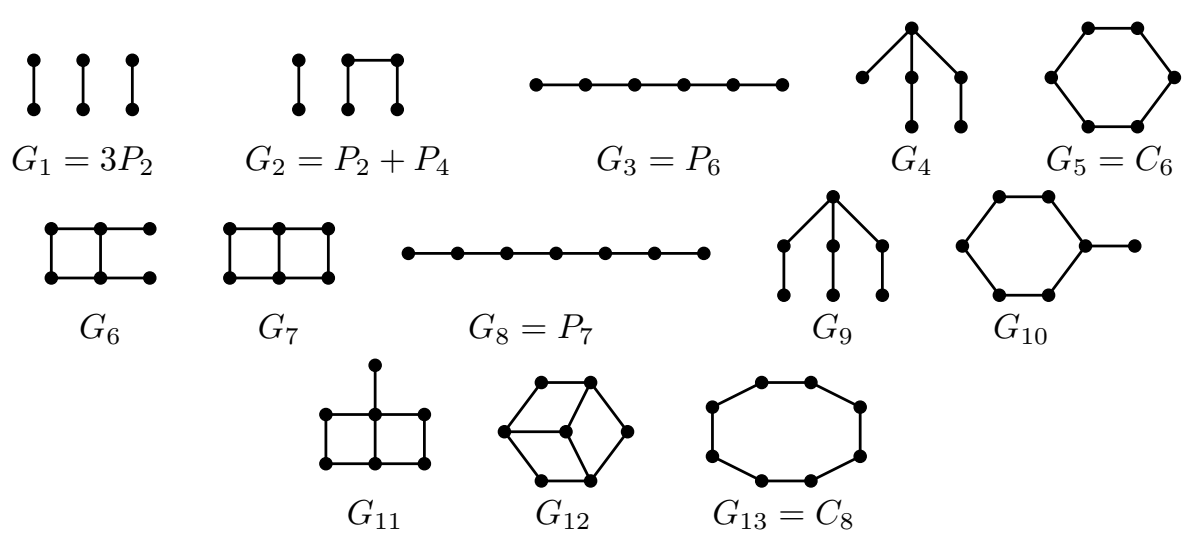

Figure 3: All strongly reduced bipartite graphs of rank 6 .

Theorem 5.1. Let $G$ be a strongly reduced bipartite graph. Then $\operatorname{rank}(G)=6$ if and only if $G$ is one of the graphs which are shown in Figure 3.

Proof. Let $X$ and $Y$ be the parts of $G$ and $B=B(G)$. Since $\operatorname{rank}(G)=6$, by Theorem 2.1, $\operatorname{rank}(B)=3$. We show that $|X|,|Y| \leq 4$ (that is $G$ has at most 8 vertices). Let $y, y_{1}, y_{2}, y_{3}$ be four vertices of $Y$. Since $\operatorname{rank}(B)=3$, there exist some real numbers $a, a_{1}, a_{2}$ and $a_{3}$ such that $a[y]+a_{1}\left[y_{1}\right]+a_{2}\left[y_{2}\right]+a_{3}\left[y_{3}\right]=0$. We claim that all the numbers $a, a_{1}, a_{2}$ and $a_{3}$ are non-zero. If two coefficients are zero, for example $a_{2}=a_{3}=0$, then $N(y)=N\left(y_{1}\right)$, a contradiction (because $G$ is reduced). If one of the coefficients is zero, say $a=0$, then as we see in the proof of Theorem 4.2, one of the following holds: 
$\left[y_{1}\right]=\left[y_{2}\right]+\left[y_{3}\right]$ or $\left[y_{2}\right]=\left[y_{1}\right]+\left[y_{3}\right]$ or $\left[y_{3}\right]=\left[y_{1}\right]+\left[y_{2}\right]$, a contradiction $($ since $G$ is neighbor-reduced). Thus the claim is proved.

Suppose that $\operatorname{deg}(y) \geq \operatorname{deg}\left(y_{1}\right) \geq \operatorname{deg}\left(y_{2}\right) \geq \operatorname{deg}\left(y_{3}\right)$. One can see that $N(y) \nsubseteq$ $N\left(y_{i}\right)$, for $i=1,2,3$. Let

$$
[y]=\alpha\left[y_{1}\right]+\beta\left[y_{2}\right]+\gamma\left[y_{3}\right], \text { where } \alpha, \beta, \gamma \neq 0 .
$$

Since $N(y) \backslash N\left(y_{1}\right) \neq \varnothing$, by Equation (5.1) we obtain $\beta l_{1}+\gamma l_{2}=1$, where $l_{1}, l_{2} \in$ $\{0,1\}$. This shows that $\beta=1$ or $\gamma=1$ or $\beta+\gamma=1$. Similarly, since $N(y) \nsubseteq N\left(y_{i}\right)$ for $i=2,3$, we have $\alpha=1$ or $\gamma=1$ or $\alpha+\gamma=1$ and $\alpha=1$ or $\beta=1$ or $\alpha+\beta=1$. It is not hard to see that the multiset $\{\alpha, \beta, \gamma\}$ is one of the following:

$$
\left\{\frac{1}{2}, \frac{1}{2}, \frac{1}{2}\right\},\{1,1,1\},\{-1,1,1\},\{-2,1,1\},\{-1,1,2\},\{-1,-1,1\} .
$$

Therefore $[y]$ satisfying in one of the following equations:

$$
\begin{aligned}
& \mathbf{L}_{\mathbf{1}}: 2[y]=\left[y_{1}\right]+\left[y_{2}\right]+\left[y_{3}\right] . \quad \mathbf{L}_{\mathbf{2}}:[y]=\left[y_{1}\right]+\left[y_{2}\right]+\left[y_{3}\right] . \quad \mathbf{L}_{\mathbf{3}}:[y]=\left[y_{1}\right]+\left[y_{2}\right]-\left[y_{3}\right] . \\
& \mathbf{L}_{\mathbf{4}}: {[y]=\left[y_{1}\right]-\left[y_{2}\right]+\left[y_{3}\right] . \quad \mathbf{L}_{\mathbf{5}}:[y]=-\left[y_{1}\right]+\left[y_{2}\right]+\left[y_{3}\right] . \quad \mathbf{L}_{\mathbf{6}}:[y]=\left[y_{1}\right]+\left[y_{2}\right]-2\left[y_{3}\right] . } \\
& \mathbf{L}_{\mathbf{7}}:[y]=\left[y_{1}\right]-2\left[y_{2}\right]+\left[y_{3}\right] . \mathbf{L}_{\mathbf{8}}:[y]=-2\left[y_{1}\right]+\left[y_{2}\right]+\left[y_{3}\right] . \quad \mathbf{L}_{\mathbf{9}}:[y]=2\left[y_{1}\right]+\left[y_{2}\right]-\left[y_{3}\right] . \\
& \mathbf{L}_{\mathbf{1 0}}:[y]=2\left[y_{1}\right]-\left[y_{2}\right]+\left[y_{3}\right] . \mathbf{L}_{\mathbf{1 1}}:[y]=\left[y_{1}\right]+2\left[y_{2}\right]-\left[y_{3}\right] . \mathbf{L}_{\mathbf{1 2}}:[y]=-\left[y_{1}\right]+2\left[y_{2}\right]+\left[y_{3}\right] . \\
& \mathbf{L}_{\mathbf{1 3}}:[y]=\left[y_{1}\right]-\left[y_{2}\right]+2\left[y_{3}\right] . \mathbf{L}_{\mathbf{1 4}}:[y]=-\left[y_{1}\right]+\left[y_{2}\right]+2\left[y_{3}\right] . \mathbf{L}_{\mathbf{1 5}}:[y]=\left[y_{1}\right]-\left[y_{2}\right]-\left[y_{3}\right] . \\
& \mathbf{L}_{\mathbf{1 6}}:[y]=-\left[y_{1}\right]+\left[y_{2}\right]-\left[y_{3}\right] . \quad \mathbf{L}_{\mathbf{1 7}}:[y]=-\left[y_{1}\right]-\left[y_{2}\right]+\left[y_{3}\right] .
\end{aligned}
$$

By multiplying the vector $j$ (all components are 1) to both sides of Equation (5.1) we conclude that

$$
\operatorname{deg}(y)=\alpha \operatorname{deg}\left(y_{1}\right)+\beta \operatorname{deg}\left(y_{2}\right)+\gamma \operatorname{deg}\left(y_{3}\right) .
$$

Suppose that $y_{1}, y_{2}, y_{3}$ have the minimum degree among all vertices of $Y$. Let $\operatorname{deg}\left(y_{1}\right) \geq \operatorname{deg}\left(y_{2}\right) \geq \operatorname{deg}\left(y_{3}\right)$. By Equation (5.2) the cases $L_{8}, L_{15}, L_{16}, L_{17}$ cannot happen. On the other hand one can see that the cases $L_{7}, L_{9}, L_{10}, L_{11}$ can not occur. For example suppose that $L_{7}$ holds. So there exists a vertex $y \neq y_{1}, y_{2}, y_{3}$ in $Y$ such $[y]=\left[y_{1}\right]-2\left[y_{2}\right]+\left[y_{3}\right]$. This shows that $N\left(y_{2}\right) \subseteq N\left(y_{1}\right) \cap N\left(y_{3}\right)$. Since $\operatorname{deg}\left(y_{2}\right) \geq \operatorname{deg}\left(y_{3}\right), N\left(y_{2}\right)=N\left(y_{3}\right)$, a contradiction (because $G$ is reduced).

Now we claim that $|Y| \leq 4$. By contradiction let $|Y| \geq 5$. Assume that $y_{1}, y_{2}, y_{3}$ have the minimum degree among all vertices of $Y$ and $\operatorname{deg}\left(y_{1}\right) \geq \operatorname{deg}\left(y_{2}\right) \geq \operatorname{deg}\left(y_{3}\right)$. Suppose that $y$ and $y_{0}$ are two vertices of $Y$ (distinct from $y_{1}, y_{2}, y_{3}$ ). Thus $[y]$ and $\left[y_{0}\right]$ are satisfying in one of the equations $L_{1}, L_{2}, L_{3}, L_{4}, L_{5}, L_{6}, L_{12}, L_{13}, L_{14}$. We show that it is impossible. For example suppose that $[y]$ satisfying in the equation $L_{i}$ and $y_{0}$ in $L_{j}$. Clearly, $i \neq j$. For instance let $i=3$ and $j=4$. That is $[y]=\left[y_{1}\right]+\left[y_{2}\right]-\left[y_{3}\right]$ and $\left[y_{0}\right]=\left[y_{1}\right]-\left[y_{2}\right]+\left[y_{3}\right]$. Thus $[y]+\left[y_{0}\right]=2\left[y_{1}\right]$, a contradiction (because every three columns of $B$ is independent). As other manner let $i=1$ and $j=2$. Thus $\left[y_{0}\right]=$ $-2[y]+2\left[y_{2}\right]+2\left[y_{3}\right]$, a contradiction ( because it does not appear in equations $L_{1}, \ldots, L_{17}$ ). As a last manner let $i=12$ and $j=14$. The condition $L_{12}$ implies that $N\left(y_{2}\right) \subseteq N\left(y_{1}\right)$ and $N\left(y_{2}\right) \cap N\left(y_{3}\right)=\varnothing$. On the other hand $L_{14}$ shows that $N\left(y_{3}\right) \subseteq N\left(y_{1}\right)$. Since $G$ is neighbor-reduced, $N\left(y_{1}\right) \neq N\left(y_{2}\right) \cup N\left(y_{3}\right)$. Let $x \in N\left(y_{1}\right) \backslash N\left(y_{2}\right) \cup N\left(y_{3}\right)$. By the equality $[y]=-\left[y_{1}\right]+2\left[y_{2}\right]+\left[y_{3}\right]$, we find that the component correspondences to $x$ in $[y]$ is -1 , a contradiction. Similarly one can check the other value for $i$ and $j$, where 
$i, j \in\{1,2,3,4,5,6,12,13,14\}$. The claim is proved. That is $|Y| \leq 4$. Similarly $|X| \leq 4$. Thus $G$ has at most 8 vertices. One can see that all of the strongly reduced graphs of rank 6 and with at most 8 vertices are given in Figure 3. The proof is complete.

Remark 5.2. Let $b_{n}$ and $c_{n}$ be the number of connected bipartite graphs and connected strongly reduced bipartite graphs of order $n$, respectively. Then

$$
\begin{aligned}
& b_{1}=b_{2}=b_{3}=1, b_{4}=3, b_{5}=5, b_{6}=17, b_{7}=44, b_{8}=182, \\
& c_{1}=c_{2}=1, c_{3}=0, c_{4}=1, c_{5}=0, c_{6}=5, c_{7}=5, c_{8}=36 .
\end{aligned}
$$

We note that among all connected strongly reduced bipartite graphs of order 8 , the cycle $C_{8}$ is the only graph with rank 6 and the others have rank 8.

Using Theorems 2.5 and 5.1 ( similar to proof of Theorems 3.3 and 4.3) we characterize all bipartite graphs of rank 6. See also Theorem 8.3 of [8].

Theorem 5.3. Let $G$ be a bipartite graph with no isolated vertex. Then $\operatorname{rank}(G)=6$ if and only if $G$ is obtained from one of the graph which is shown in Figure 3 with the operations $\star$ and $\oplus$.

Now we characterize all bipartite reduced graphs of rank 6 .

Theorem 5.4. Let $G$ be a reduced bipartite graph. Then $\operatorname{rank}(G)=6$ if and only if $G$ is one of the graphs which are shown in Figurer 4.

Proof. It is easy to check that all graphs of Figure 4 are reduced of rank 6 . Thus it remains to prove the other part. Let $G$ be a reduced bipartite graph of rank 6 . If $G$ is strongly reduced, then by Theorem 5.1, $G$ is one of the graphs $G_{1}, \ldots, G_{13}$ which are shown in Figure 3. Otherwise, by Theorem 5.3, $G$ is obtained from one of the graphs $G_{1}, \ldots, G_{13}$ by the operations $\star$ and $\oplus$. Since $G$ is reduced, $G$ is obtained only by the operation $\oplus$ from $G_{1}, \ldots, G_{13}$ (note that if $H$ is a non-reduced graph, then for any independent set $S$ of $H$, $H \star S$ is also non-reduced).

Thus to obtain $G$ it is sufficient to apply the operation $\oplus$ on the graphs $G_{1}, \ldots, G_{13}$. Since there is no non-adjacent vertices $u$ and $v$ in $G_{5}, G_{6}, G_{7}, G_{11}, G_{12}$ such that $N(u) \cap$ $N(v)=\varnothing$, we can not apply $\oplus$ on the graphs $G_{5}, G_{6}, G_{7}, G_{11}, G_{12}$.

For the other graphs, one can see the following:

1) The graphs $G_{1,1}, G_{1,2}, G_{1,3}, G_{1,4}, G_{1,5}, G_{1,6}, G_{1,7}$ and $G_{1,8}$ are obtained from $G_{1}$.

2) The graphs $G_{2,1}, G_{2,2}, G_{2,3}$ and $G_{2,4}$ are obtained from $G_{2}$.

3) The graph $G_{3,1}$ is obtained from $G_{3}$.

4) The graph $G_{4,1}$ is obtained from $G_{4}$.

5) The graphs $G_{8,1}, G_{8,2}, G_{8,3}$ and $G_{8,4}$ are obtained from $G_{8}$.

6) The graphs $G_{9,1}, G_{9,2}$ and $G_{9,3}$ that are isomorphic to $G_{1,4}, G_{1,6}$ and $G_{1,8}$, respectively, are obtained from $G_{9}$.

7) The graph $G_{10,1}$ is obtained from $G_{10}$.

8) The graph $G_{13,1}$ is obtained from $G_{13}$. 
Therefore we obtain 20 reduced graphs from $G_{1}, G_{2}, G_{3}, G_{4}, G_{8}, G_{9}, G_{10}$ and $G_{13}$. Since $G_{1}, \ldots, G_{13}$ are also reduced, there are exactly 33 bipartite reduced graphs of rank 6 ( see Figure 4).

Similar to other theorems like Theorem 5.3 we can prove one of the main result of this paper.

Theorem 5.5. Let $G$ be a bipartite graph with no isolated vertex. Then $\operatorname{rank}(G)=6$ if and only if $G$ obtained from one of the graphs which are shown in Figurer 4 with the operation $\star$.

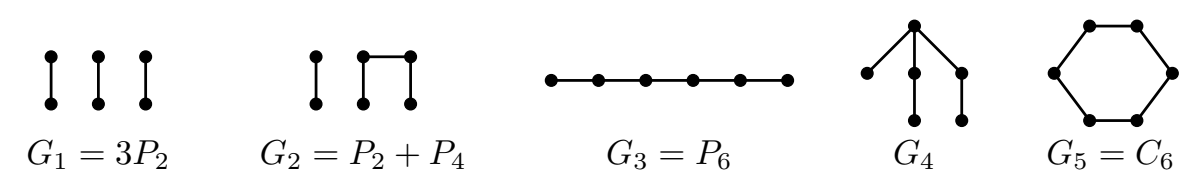

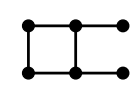

$G_{6}$

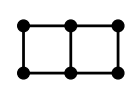

$G_{7}$

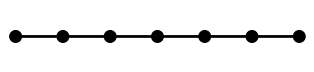

$G_{8}=P_{7}$
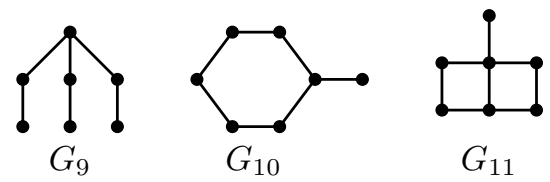

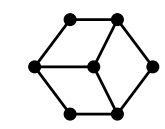

$G_{12}$

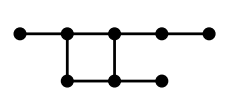

$G_{1,4}$

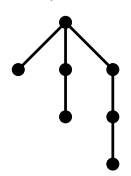

$G_{2,1}$

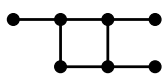

$G_{4,1}$

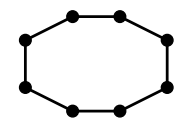

$G_{13}=C_{8}$
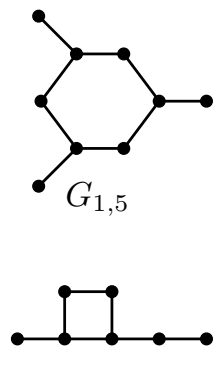

$G_{2,2}$

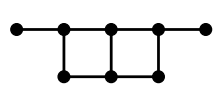

$G_{8,1}$

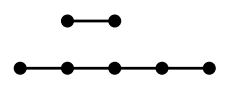

$G_{1,1}=P_{2}+P_{5}$
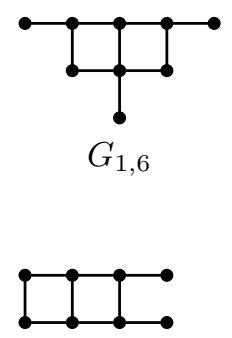

$G_{2,3}$

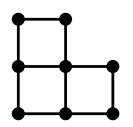

$G_{8,2}$

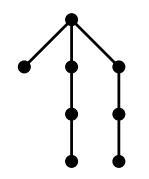

$G_{1,2}$
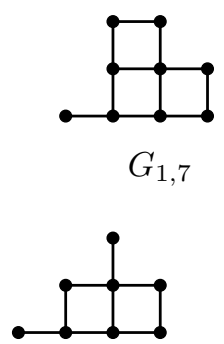

$G_{2,4}$

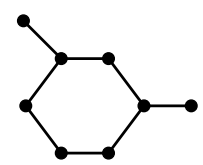

$G_{8,3}$

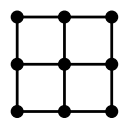

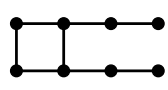

$G_{1,3}$
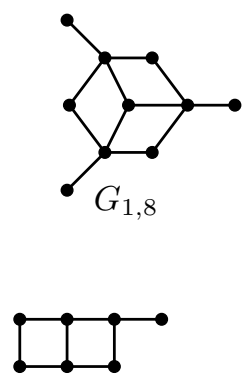

$G_{3,1}$

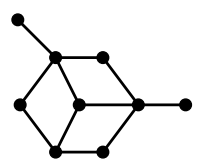

$G_{8,4}$

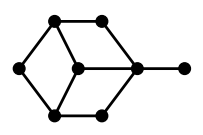

$G_{10,1}$
$G_{13,1}$

Figure 4: All reduced bipartite graphs of rank 6 . 


\section{References}

[1] G. J. Chang, L.-H. Huang and H.-G. Yeh, A characterization of graphs with rank 4, Linear Algebra Appl. 434 (2011), 1793-1798, doi:10.1016/j.laa.2010.09.040.

[2] G. J. Chang, L.-H. Huang and H.-G. Yeh, A characterization of graphs with rank 5, Linear Algebra Appl. 436 (2012), 4241-4250, doi:10.1016/j.laa.2012.01.021.

[3] D. Cvetković, P. Rowlinson and S. Simić, An introduction to the theory of graph spectra, volume 75 of London Mathematical Society Student Texts, Cambridge University Press, Cambridge, 2010 .

[4] D. M. Cvetković, M. Doob and H. Sachs, Spectra of graphs, Theory and application, volume 87 of Pure and Applied Mathematics, Academic Press, Inc. [Harcourt Brace Jovanovich, Publishers], New York-London, 1980.

[5] Y.-Z. Fan and K.-S. Qian, On the nullity of bipartite graphs, Linear Algebra Appl. 430 (2009), 2943-2949, doi:10.1016/j.laa.2009.01.007.

[6] A. Farrugia and I. Sciriha, The main eigenvalues and number of walks in self-complementary graphs, Linear Multilinear Algebra 62 (2014), 1346-1360, doi:10.1080/03081087.2013.825959.

[7] A. Kotlov and L. Lovász, The rank and size of graphs, J. Graph Theory 23 (1996), 185-189, doi:10.1002/(SICI)1097-0118(199610)23:2〈185::AID-JGT9〉3.3.CO;2-X.

[8] I. Sciriha, On the rank of graphs, in: Combinatorics, graph theory, and algorithms, Vol. I, II (Kalamazoo, MI, 1996), New Issues Press, Kalamazoo, MI, pp. 769-778, 1999.

[9] L. Wang, Y. Fan and Y. Wang, The triangle-free graphs with rank 6, J. Math. Res. Appl. 34 (2014), 517-528. 